\title{
TELEOLOGICAL COMPUTER GRAPHICS MODELING
}

\author{
Alan H. Barr \\ Computer Graphics Laboratory \\ California Institute of Technology
}

\begin{abstract}
Computer graphics consists of two main activities: modeling, which is the science of making mathematical abstractions of objects, and rendering or visualization, in which images are made from the mathematical abstractions. In this talk, teleological modeling is presented, a developing approach for creating abstractions and mathematical representations of physically realistic time-dependent objects. In this approach, geometric constraint properties, mechanical properties of objects, the parameters representing an object, and the control of the object are incorporated into a single conceptual framework.

A teleological model incorporates time-dependent goals of behavior or "purpose" as the primary abstraction and representation of what the object is; the term is derived from the Greek word "telos", for end, goal, or purpose. In this way, a teleological model usually implies geometry rather than prescribing or specifying geometry directly. A teleological implementation takes a geometrically "incomplete" specification of the motion, position, and shape of an object, and produces a geometrically complete description of the object's shape and behavior as a function of time. Intuitively, it is a representation which calculates the object's behavior from what the object is "supposed" to do.

Teleological modeling techniques may be suitable for consideration in computer vision algorithms, by extending the current notions about how to make mathematical representations of objects. Teleological descriptions can produce compact representations for many of the physically derivable quantities controlling the shapes, combining-operations, and constraints which govern the formation and motion of objects.
\end{abstract}

